\title{
Heparin-free extracorporeal membrane oxygenation in a patient with severe pulmonary contusions and bronchial disruption
}

\author{
Kyoung Min Ryu', Sung Wook Chang ${ }^{1,2}$ \\ ${ }^{1}$ Department of Thoracic and Cardiovascular Surgery, ${ }^{2}$ Trauma Center, Dankook University Hospital, \\ Cheonan, Korea
}

Pulmonary contusion complicated with endobronchial hemorrhage is potentially life-threatening, particularly in patients with tracheobronchial tree disruption and severe airway bleeding after blunt trauma, and pose a high mortality risk. In such cases, extracorporeal membrane oxygenation (ECMO) can be used as a salvage treatment modality. However, the use of ECMO for moribund trauma patients with respiratory failure may be limited for several reasons, such as intractable bleeding. In this case report, we describe a patient with severe bilateral pulmonary contusions with tracheobronchial tree disruption that was successfully treated using heparinfree venovenous ECMO.

Keywords Lung injury; Bronchi; Heparin; Extracorporeal membrane oxygenation
What is already known

In selected trauma patients with hemorrhage, heparin-free extracorporeal membrane oxygenation may be used successfully.

What is new in the current study

We report early decision-making and the use of heparin-free extracorporeal membrane oxygenation to support a patient with tracheobronchial tree disruption and bilateral pulmonary contusions complicated with alveolar hemorrhage due to a blunt trauma.
elSSN: 2383-4625

Received: 11 January 2018

Revised: 25 February 2018

Accepted: 25 February 2018

Correspondence to: Sung Wook Chang Department of Thoracic and Cardiovascular Surgery, Dankook University Hospital, 201 Manghyangro, Dongnam-gu, Cheonan 31116, Korea E-mail:changsw3@naver.com

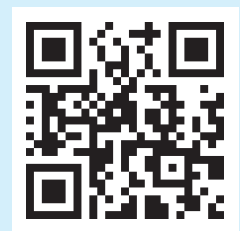

How to cite this article: Ryu KM, Chang SW. Heparin-free extracorporeal membrane oxygenation in a patient with severe pulmonary contusions and bronchial disruption. Clin Exp Emerg Med 2018;5(3):204-207.

This is an Open Access article distributed under the terms of the Creative Commons Attribution Non-Commercial License (http:// creativecommons.org/licenses/by-nc/4.0/). 


\section{INTRODUCTION}

Extracorporeal membrane oxygenation (ECMO) is a useful rescue treatment for patients with respiratory failure. However, the use of ECMO in trauma patients with bleeding has been limited because of the risk of systemic heparinization. In selected trauma patients with endobronchial hemorrhage due to severe contusion, heparin-free ECMO may be used successfully. ${ }^{1}$ In this case report, we report the use of heparin-free ECMO to support a patient with tracheobronchial tree disruption and bilateral pulmonary contusions complicated with alveolar hemorrhage due to a blunt trauma.

\section{CASE REPORT}

A 48-year-old man who had sustained traumatic hemopneumothorax after being trapped between a forklift and a truck was transferred from a local hospital after bilateral closed thoracostomy. His initial vital signs were as follows: blood pressure, 160/100 $\mathrm{mmHg}$; pulse rate, 104 beats/min; respiratory rate (RR), 32 breaths/ min; oxygen saturation, $92 \%$; body temperature, $36.5^{\circ} \mathrm{C}$; and Glasgow coma scale, 15 points.

Initial arterial blood gas analysis performed under mechanical ventilation with a fraction of inspired oxygen $\left(\mathrm{FiO}_{2}\right)$ of 0.6 showed a pH of $7.22, \mathrm{PaCO}_{2}$ of $50 \mathrm{mmHg}, \mathrm{PaO}_{2}$ of $64 \mathrm{mmHg}$, and $\mathrm{HCO}_{3}$ of $22 \mathrm{mmol} / \mathrm{L}$ Contrast-enhanced computed tomography of the chest and abdomen-pelvis revealed a grade 2 liver laceration, multiple thoracic spine fractures involving the transverse and spinous processes, bilateral multiple rib fractures, and severe bilateral lung contusions with suspicious injury of the right lower lobar bronchus. The injury severity score was 43 points (Fig. 1).

After 4 hours, continuous air leakage through the right-sided chest tube was observed. The patient's ventilatory parameters were incompatible with survival, with a $\mathrm{PaO}_{2} / \mathrm{FiO}_{2}$ ratio of 56 at $\mathrm{FiO}_{2}$ of 1.0. Venovenous ECMO (Maquet Rotaflow Centrifugal Pumps with Quadrox-D oxygenators; Maquet, Rastatt, Germany) was promptly established using the Seldinger technique. A 17-Fr arterial catheter was inserted into the right jugular vein and a 19-Fr venous catheter (Bio-medicus; Medtronic, Minneapolis, MN, USA) was inserted into the right femoral vein. Initial heparin loading was not performed. After ECMO, the ventilatory status and follow-up arterial blood gas analysis were as follows: $\mathrm{FiO}_{2}$ of $0.4, \mathrm{RR}$ of 12 breaths/min, peak inspiratory pressure of $20 \mathrm{mmHg}$, positive endexpiratory pressure of $10 \mathrm{mmHg}, \mathrm{PaO}_{2}$ of $98 \mathrm{mmHg}$, and $\mathrm{PaCO}_{2}$ 28 of $\mathrm{mmHg}$. Under ECMO, we performed a bronchoscopy to evaluate the extent of the bronchial injury and then decided to perform emergency surgery.

Surgery was performed under venovenous ECMO, with onelung ventilation and lung protection strategies implemented to keep the tidal volume below $150 \mathrm{~mL}$ and $\mathrm{FiO}_{2}$ below 0.4. A right posterolateral thoracotomy was performed to remove the large
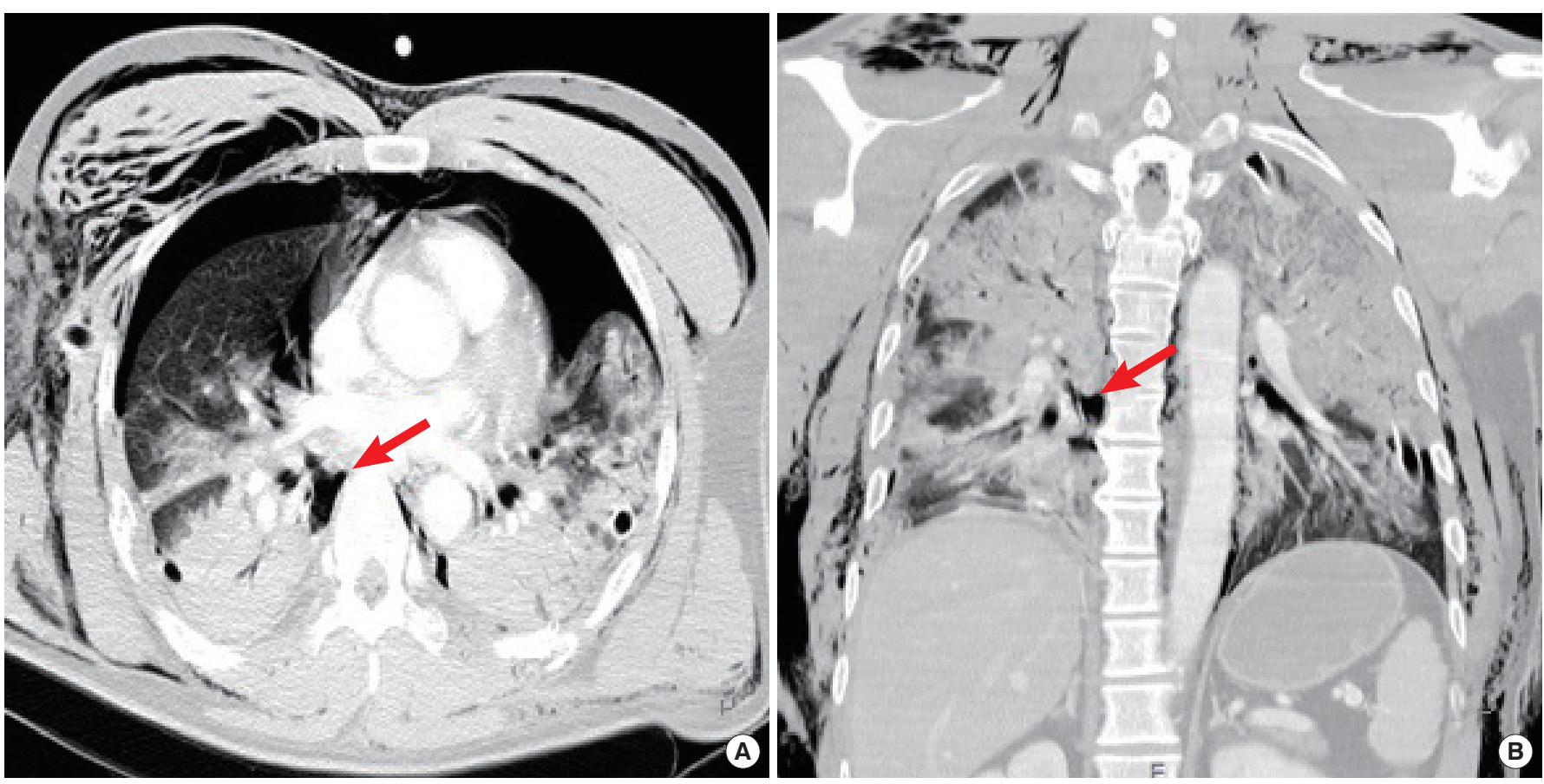

Fig. 1. Computed tomography of the chest in a 48-year-old man who sustained blunt chest trauma. $(A, B)$ There is a suspicious bronchial injury (arrow) on the right lower lobe and severe bilateral lung contusions. 

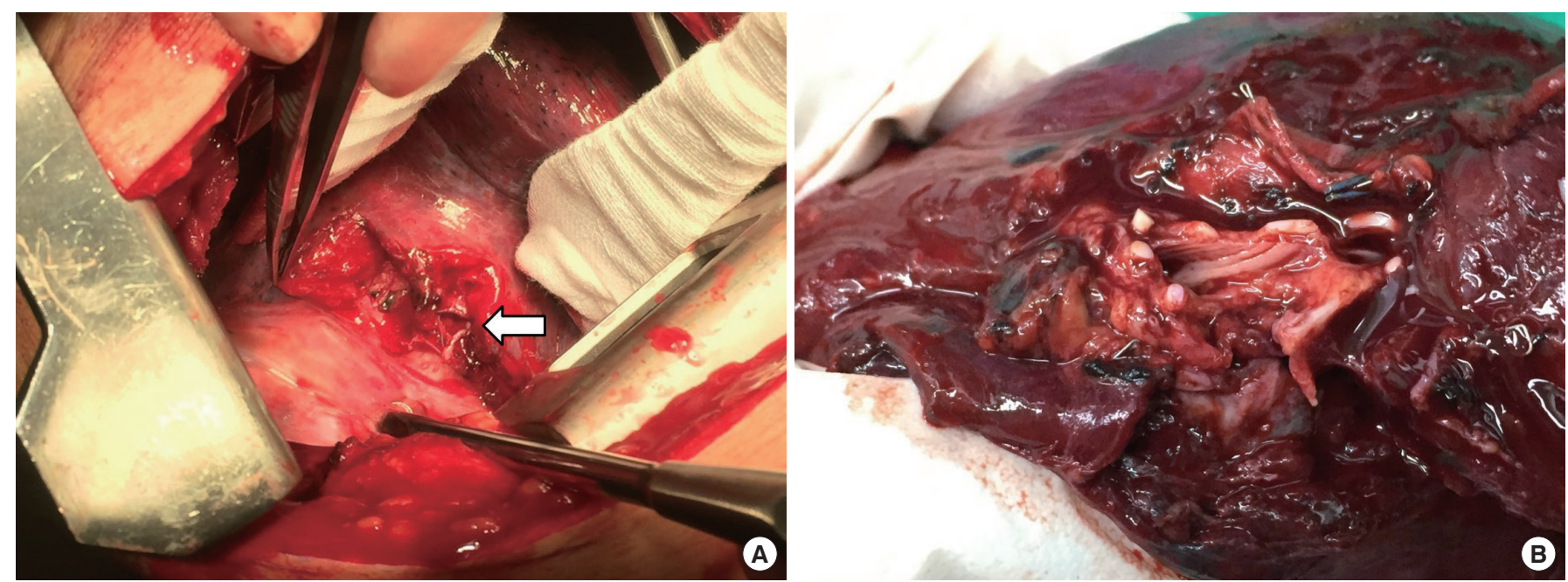

Fig. 2. Intraoperative findings in a 48-year-old man who sustained blunt chest trauma. (A) Bronchial tear and crushing injury to the right lower lobe of the lung are shown. (B) After right lower lobectomy, the specimen shows a more than $80 \%$ circumferential tear in the right lower lobar bronchus.

hematomas. A circumferential tear of more than $80 \%$ was observed in the right lower lobar bronchus, with laceration of the right lower lobe that was irreparable (Fig. 2A); hence, a right lower lobectomy was performed (Fig. 2B). Thereafter, the displaced right sixth and seventh ribs were fixed using an 8-hole straight plate (SternaLock; Biomet Microfixation, Jacksonville, FL, USA).

ECMO was maintained without anticoagulation, and meticulous lung care with suctioning of the blood clot was continued. The ECMO system was successfully removed 59 hours postoperatively. The patient was eventually weaned off mechanical ventilation on day 4 and was discharged without any complaints.

\section{DISCUSSION}

ECMO has been a proven rescue modality to treat patients with reversible respiratory failure. Currently, early initiation of ECMO is acknowledged to improve the survival of trauma patients with respiratory failure due to ventilator-associated pneumonia or systemic inflammatory response syndrome. ${ }^{1}$ However, ECMO support in patients with bronchial injury and/or severe lung contusion is not recommended. Pulmonary contusion with alveolar hemorrhage and tracheobronchial injury can be managed without ECMO through lung isolation using a double-lumen endotracheal tube and suctioning of the blood clot and strategies that range from repair to pneumonectomy. ${ }^{2-4}$ However, these treatment modalities may not be sufficient for stabilizing patients with bronchial injury complicated with bilateral lung contusions and endobronchial hemorrhage. In particular, one-lung ventilation during surgery could pose high mortality and morbidity risks because of acute lung injury, and conventional treatment might not be adequate for maintain- ing hemodynamic and respiratory stability. ${ }^{5}$ In such cases, ECMO could be a useful rescue treatment.

In general, the use of ECMO entails anticoagulation to prevent life-threatening thrombosis in the extracorporeal circuit. On the other hand, inappropriate anticoagulation may result in excessive bleeding due to consumptive coagulopathy. ${ }^{6}$ According to one report, among 15 patients, 6 had hemorrhagic complications and 4 had thromboembolic complications.' These results imply a limitation of ECMO support in moribund trauma patients with bleeding. Recently, heparin-coated circuits and oxygen membranes have been developed. According to another case report, short-term heparin-free venovenous ECMO in patients with contraindications to therapeutic anticoagulation could be an effective treatment modality without thromboembolic complications..$^{7-9}$ In this patient who presented with active bleeding, which manifested as pulmonary contusion complicated with alveolar hemorrhage, hemothorax, and liver laceration, the use of high-flow (more than $3.5 \mathrm{~L} / \mathrm{min}$ ) and short-term ECMO, instead of anticoagulation, successfully weaned him off mechanical ventilator support 59 hours postoperatively, without thromboembolic complications. As this is a case report, the use of heparin-free venovenous ECMO for patients with bleeding needs to be proven, with more cases and studies undertaken. Nevertheless, our experience with this case suggests that heparin-free ECMO may be a valuable treatment option for trauma patients with contraindications to anticoagulation.

ECMO is an invasive procedure with significant risks for complications, such as thrombosis and hemorrhage. In addition, ECMO support may not be the first treatment option in patients with bronchial injury or traumatic lung contusion with alveolar hem- 
orrhage, and its use is even contested in injured, bleeding patients. However, in a patient with severe traumatic bronchial injury and alveolar hemorrhage with intractable hypoxemia and hypercapnia, ECMO merits consideration and may be key to survival in this situation.

\section{CONFLICT OF INTEREST}

No potential conflict of interest relevant to this article was reported.

\section{REFERENCES}

1. Bosarge PL, Raff LA, McGwin G Jr, et al. Early initiation of extracorporeal membrane oxygenation improves survival in adult trauma patients with severe adult respiratory distress syndrome. J Trauma Acute Care Surg 2016;81:236-43.

2. Kabon B, Waltl B, Leitgeb J, Kapral S, Zimpfer M. First experience with fiberoptically directed wire-guided endobronchial blockade in severe pulmonary bleeding in an emergency setting. Chest 2001;120:1399-402.
3. Lee SH, Kwon JB, Choi SH. Surgical treatment of tracheobronchial injury due to blunt trauma. Korean J Thorac Cardiovasc Surg 1996;29:208-12.

4. Hwang JJ, Kim YJ, Cho HM, Lee TY. Traumatic tracheobronchial injury: delayed diagnosis and treatment outcome. Korean J Thorac Cardiovasc Surg 2013:46:197-201.

5. Della Rocca G, Coccia C. Acute lung injury in thoracic surgery. Curr Opin Anaesthesiol 2013;26:40-6.

6. Oliver WC. Anticoagulation and coagulation management for ECMO. Semin Cardiothorac Vasc Anesth 2009;13:154-75.

7. Muellenbach RM, Kredel M, Kunze E, et al. Prolonged heparinfree extracorporeal membrane oxygenation in multiple injured acute respiratory distress syndrome patients with traumatic brain injury. J Trauma Acute Care Surg 2012;72:1444-7.

8. Biscotti $M$, Gannon WD, Abrams D, et al. Extracorporeal membrane oxygenation use in patients with traumatic brain injury. Perfusion 2015;30:407-9.

9. Lamarche $Y$, Chow B, Bedard A, et al. Thromboembolic events in patients on extracorporeal membrane oxygenation without anticoagulation. Innovations (Phila) 2010;5:424-9. 